\title{
Combination Influenza Therapy with Clarithromycin-Naproxen-Oseltamivir Superior to Oseltamivir Alone
}

As we enter the influenza season, Ivan et al. (1) are reporting in Chest that oseltamivir-clarithromycin-naproxen combination for treatment of serious influenza results in reduced mortality, less frequent ICU admission, and shorter hospital-stay compared to oseltamivir alone. From February to April 2015, the authors conducted a prospective open-label randomized-controlled trial. Adult patients hospitalized for $A(H 3 N 2)$ influenza were randomly assigned to a 2-day combination of clarithromycin $500 \mathrm{mg}$, naproxen $200 \mathrm{mg}$ and oseltamivir $75 \mathrm{mg}$ twice daily, followed by 3 days of oseltamivir; or oseltamivir $75 \mathrm{mg}$ twice daily for 5 days as control (1:1). Among the 217 influenza A(H3N2) patients enrolled, 107 were randomly assigned to the combination treatment. Ten patients succumbed during the 30-day follow-up. The combination treatment was associated with lower 30 -day mortality $(p=0.01)$, less frequent ICU/HDU admission $(p<0.001)$, and shorter hospital-stay $(p<0.0001)$. Multivariate analysis showed that combination treatment was the only independent factor associated with lower 30day mortality $(p=0.04)$. The authors advised further study on the antiviral and immunomodulatory effects of this combination treatment, but those caring for severely ill patients with influenza might wish to consider combination therapy since all these drugs are available.

Richard A. Robbins, MD

Editor, SWJPCC

\section{Reference}

1. Hung IF, To KK, Chan JF, et al. Efficacy of clarithromycin-naproxenoseltamivir combination in the treatment of patients hospitalized for influenza $\mathrm{A}(\mathrm{H} 3 \mathrm{~N} 2)$ infection: an open-label, randomized controlled, phase $2 \mathrm{~b} / 3$ trial. Chest. 2016 Nov 21. [Epub ahead of print] [CrossRef] [PubMed] 